\title{
Measurement of residual thickness using scatterometry
}

David Fuard*, Corinne Perret, Vincent Farys, Cécile Gourgon, Patrick Schiavone

Laboratoire des Technologies de la Microélectronique CNRS, c/o CEA Grenoble, 17 rue des Martyrs, 38054 Grenoble Cedex 09, France

* fuardda@chartreuse.cea.fr

\begin{abstract}
Nano-Imprint Lithography (NIL) processes have the characteristic that a residual resist layer is always present between the nano-imprinted features. This residual resist layer must be removed to obtain usable resist masks for pattern transfer. As this resist layer is removed using oxygen-based plasma processes, the residual thickness non uniformity translates into feature width dispersion. Thus, the uniformity of this residual thickness after imprint remains an important issue for Nano-Imprint Lithography and a reliable metrology procedure is required for.
\end{abstract}

At present, the standard measurement method is based on Scanning Electron Microscopy (SEM) cross-section, which is destructive, time-consuming, and may sometimes provide only moderate accuracy. The work presented here will assess and show the interest of scatterometry, which is a non destructive optical method of metrology that can be easily applied to NIL.

This measurement procedure exhibits very good accuracy on the $2 \mathrm{D}$-feature geometry determination, especially for residual thickness. Scatterometry also eases time-consuming studies like residual thickness measurement at the local scale or at the wafer scale. Moreover, this paper shows that the imprint uniformity studies provide very interesting information on the mold deformation. 
Keywords: Nano-Imprint Lithography, NIL, embossing, scatterometry, MMFE, RCWT, RCWA, residual thickness.

\section{Introduction}

The Nano-Imprint Lithography (NIL) consists in embossing stamps into heated resist. After the embossing process, a residual polymer layer of thickness $\mathrm{hr}$ is always present at the bottom of the imprinted features. This residual layer must be removed to obtain a usable resist mask for pattern transfer into the underlying substrate. The residual resist thickness (RRT) is removed using oxygen-based plasma processes. Unfortunately, after the imprint process, the RRT dispersion can be very large on the wafer (from $20 \mathrm{~nm}$ to $200 \mathrm{~nm}$ ). As that dispersion translates into feature width dispersion (mainly because of the use of long over-etch times combined with micro-loading effects ${ }^{1}$ ), uniform RRT on the whole wafer remains one of the most important issues for all NIL techniques. The measurement of the RRT represents one main challenge towards a better understanding of the RRT dispersion in the NIL process.

The standard residual thickness measurement method is based on SEM cross-section (SEMcs), which is destructive and very time-consuming. ${ }^{2}$ The SEM electron beam can also induce a RRT distortion. This method suffers from a combination of several major drawbacks that can rule this technique out for intensive studies. This emphasizes the need for another reliable metrology procedure. Because of its sensitivity, speed, low cost and capability of in-line measurement, optical metrology still remains more than an alternative tool for the determining of transparent material thickness and microelectronic feature size. Among all of these, scatterometry has gained recognition as one of the top technologies for $\mathrm{CD}$ metrology as well as for process control of feature characteristics below $0.1 \mu \mathrm{m}^{3}$ It has been shown to provide reliable dimensional measurements and has the capability to bring information on the 
feature profile and even on the underlying layers ${ }^{4,5}$. This technique is based on the analysis of the diffraction of light from periodic patterns (2D or $3 \mathrm{D})$.

The scatterometric analysis can best be defined to consist of two steps. First, the diffracted light fingerprint is measured. As the phase information is present in the diffraction signatures, scatterometry may achieve high measurement precision of lines much smaller than the incident light wavelength. The diffracted pattern is very sensitive to the shape and dimensional parameters of the diffracting structure. It is used to characterize the structure itself. ${ }^{6,7}$ In addition to the period of the structure, the width and the height of the resist line, the slope of the features sidewalls, and the thicknesses of several underlying film layers can also be measured via the scatter pattern. However, this requires measuring a number of lines at once. This number is determined by the size of the incident light spot. The result is an average value, which is less susceptible to small line-to-line variations than a single SEM measurement.

The second step, the inverse problem, consists of determining the shape of the lines of the periodic structure which diffracts the incident light. To solve the inverse problem, the grating shape is parameterized ${ }^{8}$, and a parameter space is defined by allowing each grating shape parameter to vary over a chosen range. A rigorous diffraction model ${ }^{9}$ based on a modal method (MMFE - Modal Method by Fourier Expansion ${ }^{10}$, or RCWT is used to calculate the diffracted light fingerprint from each grating in the parameter space. This vector differential technique uses Maxwell's equations with appropriate boundary conditions at all interfaces, in order to solve the electric fields in any region of the sample. A complete overview of this rigorous theory and its scatterometric application are set out in reference 9. The optimization step consists either of using a library-based methodology, ${ }^{11}$ or of using standard optimization routines such as the Levenberg-Marquardt algorithm ${ }^{12,13}$ for structure size extraction. The best 
match between the experimental scatter signature and theoretically generated ones is sought by minimizing the absolute mean square error between these signatures.

Unlike top view SEM or fast Atomic Force Microscope, which are not usable in determining RRT, scatterometry suits very well for the residual thickness evaluation. The main drawback of this technique is that it not able to measure the characteristics of non-periodic patterns. In addition, scatterometry is an indirect method that cannot be expected to measure feature shape outside of the domain in which they have been planed to be looked for. This is a typical issue of most of the inverse problem strategies. However, a poor match between an experimental scatter signature and the theoretically computed one warns about the occurrence of a poor quality output. For example, the mismatch can be due to an unexpected shape of the measured feature that does not fit into the planed parameter space.

The first aim of this work was to verify the well-tried capability of scatterometry for the determining of RRT uniformity after NIL process. Comparisons between scatterometric and SEM residual thickness measurements were first performed to assess the scatterometry accuracy. RRT measurements were also conducted across a grating to show the capability for fast RRT measurements by scanning through an imprinted chip-area with a fine resolution of few microns. The results show that scatterometry provides very accurate residual thickness measurements, and that, easy and fast measurements of features characteristics can be carried out at large-scale. These results also provide additional information such as mold deformation at the grating zone edge.

\section{Experimental Setup}

In our experiments, the NIL stamps consist of $200 \mathrm{~mm}$ silicon wafers, on which patterns are designed by standard $193 \mathrm{~nm}$ lithography. After the resist development, the patterns are 
transferred into the silicon using High Density Plasma etching. The silicon etch depth is $500 \mathrm{~nm}$. After etching and resist stripping, molds are covered with a fluoro-silaned (perfluorooctyltrichlorosilane) anti-sticking layer. We used a mold layout designed with chips of $21 \mathrm{~mm}$ in width and $21 \mathrm{~mm}$ in length, repeated over the wafer. This allows studying nano-imprint uniformity and edge effects at the wafer and at the chip levels. Each chip contains 24 linegrating of $1.5 \mathrm{~mm} \times 1.5 \mathrm{~mm}$ in surface and 4 line-grating $3 \mathrm{~mm} \times 4 \mathrm{~mm}$ in surface. The line widths vary from $200 \mathrm{~nm}$ to $1.5 \mu \mathrm{m}$, with various pitches. Here, we will only present the preliminary results on the four $3 \mathrm{~mm} \times 4 \mathrm{~mm}$ gratings listed in Table 1 .

The samples consisted of photo-resist patterns obtained by one-step embossing of full $200 \mathrm{~mm}$ wafers using an EVG 520HE press. The initial resist stack was made up of $200 \mathrm{~nm}$ Sumitomo NEB22 resist on top of a reticulated $50 \mathrm{~nm}$ resist layer (baked above its reticulation temperature, at $180^{\circ} \mathrm{C}$ during 5 minutes). This bottom layer acts as an embossing stop during imprint. The upper NEB22 film is a high resolution chemically amplified spin-on resist designed for e-beam lithography. It was annealed at $110^{\circ} \mathrm{C}$ on a hot plate during 2 minutes, in order to remove a fraction of the residual solvent. The resulting resist thickness is of $250 \mathrm{~nm}$ in total. The NEB22 glass temperature $\mathrm{T}_{\mathrm{g}}$ was found to be close to $80^{\circ} \mathrm{C}$, and the printing conditions were $50^{\circ} \mathrm{C}$ above $\mathrm{T}_{\mathrm{g}}$ under vacuum $(1 \mathrm{~Pa})$. These conditions enable a suitable resist viscosity and avoid forming Saffman-Taylor instabilities ${ }^{14}$. More details concerning our NIL process are reported in reference 15

For scatterometry, we used a KLA 1280SE spectroscopic ellipsometer (wavelengths ranging from $250 \mathrm{~nm}$ to $1250 \mathrm{~nm}$ ) with a $20 \mu \mathrm{m}$-diameter spot, associated to an automated wafer stage. All measurements over the wafer are carried out automatically. Our scatterometric analysis is based on the S1 and S2 parameters, which are a combination of the standard ellipsometric angles $\psi$ and $\Delta(S 1=\cos (2 \psi)$ and $S 2=\sin (2 \psi) \cdot \cos (\Delta))$. They present the advantages of being bounded and of being the parameters directly measured by the ellipsometer. The 
MMFE truncation order is optimized for each feature pitch (cf. Table 1) in order to avoid useless computation time when convergence is reached. We used about 30 wavelengths per spectrum; this has proved to provide stable feature parameters determination. The spectra for both experimental and theoretical signatures were varied from $1.5 \mathrm{eV}$ to $4.5 \mathrm{eV}$. In order to speed up the analysis and to make the optimization more robust, all the comparisons have been carried out by using a combination of small libraries of computed spectra and a Levenberg-Marquardt optimization scheme successively. A pure library-based strategy, although the generation of the signatures needs to be performed only once, can be very time consuming, especially when the number of fitted parameters increases. The optimization approach is also more flexible than the library and also allows better computational resolution, which is not limited by the granular aspect of the library.

The residual error, or normalized mean square error (NMSE), between an unknown experimental signature and a computed signature is defined as: $\frac{1}{N} \sum_{i=1}^{N}\left(X_{e}\left(\lambda_{i}\right)-X_{c}\left(\lambda_{i}\right)\right)^{2}$, where $X_{c}\left(\lambda_{i}\right)$ is the $\mathrm{i}^{\text {th }}$ data point of the computed signature, $X_{e}\left(\lambda_{i}\right)$ is the $\mathrm{i}^{\text {th }}$ data point of the experimental one. $\mathrm{N}$ is the number of data points in each spectrum. The feature characteristics (RRT, width, height and sidewall slope of the imprinted features) associated with the closest match are taken to be the parameters of the structure. In order to confirm that the parameter space used is valid for the samples being measured, it is important to check the residual error. A measurement result is experimentally considered to be acceptable if the NMSE is smaller than about $2 \cdot 10^{-2}$. However, this particular error threshold should not be considered to be a hard rule. NMSE values could be even $50 \%$ higher, though still acceptable, depending on the samples being measured and how well their physical structure (including profile shape) has been accounted for in the model. Further work is still needed in order to rigorously quantify the measurement accuracy. 
In the next section, the scatterometric results are compared with SEM measurements, which are conducted on Hitachi SEM 4000. The samples were covered by $2 \pm 1 \mathrm{~nm}$ of gold-palladium alloy to avoid electronic charging during SEM cross-section view acquisitions.

\section{Results}

The main purpose of this preliminary work was to assess the accuracy and the sensitivity of scatterometry for the measurement of the resist residual layer thickness at first, but also for the other feature characteristics. The measurements were taken at the center of the $3 \mathrm{~mm} x$ $4 \mathrm{~mm}$ grating in order to avoid edge effects.

\subsection{Comparison to SEM}

Raymond et al. have already shown that scatterometry and cross-section SEM results agree quite well for the determination of resist line-width, resist height or anti-reflective coating thickness $^{4,16}$. Although we used spectroscopic ellipsometry, we performed similar comparison for residual layer thickness measurement in this study. As we know that the silicon mold features are not vertical (they show a typical sidewall slope of $80 \pm 5^{\circ}$ ), simple "square" and "trapezoidal" models were chosen to assess the scatterometry capability. These shapes are respectively represented by one and five slices in the modeling of the structures used in the rigorous electromagnetic modeling of the gratings. Figure 1 shows at the same time the result of the fit between experimental (grey solid lines) and theoretical (black dotted lines with squares and triangles for 1-slice and trapezoidal 5-slice models respectively) scatterometric spectra, and the comparison of these results with the SEM-cross-section views. Table 1 gathers the measurements of residual thicknesses by SEM-cs and scatterometry. These comparisons show that residual thicknesses obtained using scatterometry match the SEM data very well for nominal line-widths ranging from $0.35 \mu \mathrm{m}$ to $1 \mu \mathrm{m}$. A trapezoid model improves both residual thickness and geometrical parameter measurements, but increases the 
computing time. Considering that the sample plating overestimates the SEM-measured residual thicknesses of about $2 \mathrm{~nm}$, the residual thickness measurements agreement appears very good for $0.35 \mu \mathrm{m}$ and $0.5 \mu \mathrm{m}$ lines with about $1 \mathrm{~nm}$ difference, especially when using the trapezoid model. For $1 \mu \mathrm{m}$ lines, the line-widths agreement is of about $5 \mathrm{~nm}$, which remains small. In all these cases, the scatterometry residual error does not exceed $3.10^{-2}$. The other dimensional parameters of the diffracting structures are listed on Table 2 for information.

For the $1.5 \mu \mathrm{m}$ lines, the mold was not totally filled up during the imprint process, and therefore the resist lines were not well-defined. Figure 1 shows that there is a clear disagreement between SEM and scatterometry measurements of the residual thickness: the values, $119 \mathrm{~nm}$ for square model and $135 \mathrm{~nm}$ for trapezoid model, are well above the SEM-cs measurements $(67 \mathrm{~nm})$. In this case, the residual error is of $10^{-1}$ or more, thus well above the estimated "safe" value. That indicates that the chosen model is not consistent with the real pattern present on the measured wafer site. The plots reveal that a good match between scatterometry and SEM-cross-section is strongly correlated with a small residual error. Finally, residual error allows assessing the result quality and warns about potential inconsistency in the measurement.

\subsection{Measurement precision}

Despite the fact that the optimization algorithm is able to reach final residual thickness with a numerical precision well below the nanometer, this does not mean that the measurement precision on residual thickness measurement is such. In this section, we make an attempt to provide a first order value of the precision. We define the precision of residual thickness measurement from the NMSE variation as a function of the residual thickness variation of the computed signatures around the nominal. Of course in this case, the NMSE variation as a function residual layer thickness is totally due to that residual layer thickness variation. Thus, 
a reasonable estimation can be drawn by looking at the variation of the Normalized Mean Square Error versus a shift of the resist residual layer thickness for a particular feature. We also assume that the silicon and resist indices (as a function of wavelength) are perfectly known. The Figure 2 depicts these variations for $350 \mathrm{~nm}$ (a), $500 \mathrm{~nm}$ (b), and $1000 \mathrm{~nm}$ (c) nested lines (line-width and space-width are the same).

From Figure 2(a) and Figure 2(c), we infer that a residual thickness shift of $1 \mathrm{~nm}$ corresponds to residual error variations of $7.10^{-4}$ for $350 \mathrm{~nm}$ line and $3.10^{-4}$ for $1000 \mathrm{~nm}$ line. In the same way, a residual error of $10^{-2}$ corresponds to $4 \mathrm{~nm}$ and $6 \mathrm{~nm}$ shifts of residual thickness respectively. The same trends can be drawn for equal line and space gratings of 1 and $3 \mu \mathrm{m}$ period. Thus, commonly, residual error shifts of $5.10^{-4}$ and $10^{-2}$ respectively provide measurement precisions of about $1.0 \pm 0.5 \mathrm{~nm}$ and $5 \pm 1 \mathrm{~nm}$, if residual thickness mismatch is considered only. Note that the response is not linear.

In fact, the measurement precision depends on mutually dependent factors such as pattern characteristics, chosen wavelengths in the experimental spectra, experimental noise, geometric model and truncation order for instance. In any case, the experimental NMSE, which includes all error contributors listed above, is an upper bound of the error due to residual thickness only. Table 1 shows that the NMSE for $350 \mathrm{~nm}, 500 \mathrm{~nm}$ and $1000 \mathrm{~nm}$ halfpitch features respectively are $0.81 .10^{-2}, 2.8 .10^{-2}$, and $1.8 .10^{-2}$, which respectively correspond to $4 \mathrm{~nm}, 8 \mathrm{~nm}$ and $10 \mathrm{~nm}$ RRT measurement precisions according to the Figure 2 . The Table 1 also shows that these experimental NMSE values largely overestimate the errors on the residual thicknesses. The comparison to SEM values shows that the disagreement is rather in the nanometer range. This confirms that scatterometry, as regular ellipsometry, is very sensitive to thickness variations. However, further work is still needed before being able to give rigorous figures on the precision of scatterometry in the measurement of residual thickness. 


\subsection{Example of application for NIL process investigation}

We have shown that scatterometry enables residual thickness extraction. The purpose of this section is to show that scatterometry can be used to study residual thickness variation at a "local" scale. For example here, we studied how residual layer behaves close to a grating edge, nearby a flat area. As the mold was realized using positive resist, this flat area outside the grating was a non-etched zone of the silicon mold. This flat area does not need to be filled up as the nested patterns of the mold do nearby. Here, we use scatterometry to measure the residual thickness close to the chip edge by scanning from the edge to the chip center. The scan was made possible thanks to the small spot size of our ellipsometer $(20 \mu \mathrm{m})$ and to the automated wafer stage that allows for scanning the wafer with fine steps. Figure 3 shows a $0.7 \mathrm{~mm}$ scan performed at the edge of the $0.35 \mu \mathrm{m}$ nominal line-width grating with $20 \mu \mathrm{m}$ steps. Figure 3 depicts the measurement of the residual layer thickness from the edge to the center of the $0.35 \mu \mathrm{m}$ line grating zone. The abscissas are positive towards the grating center, and the origin of the $\mathrm{x}$ axis corresponds to the grating edge. The bottom curve shows the residual layer thickness along the scatterometric scan path. This residual thickness can also be viewed as the mold deformation fingerprint. This result shows that according our experimental conditions, constant residual thickness is reached out approximately $400 \mu \mathrm{m}$ away from the grating edge. The top curve plots the height of imprinted lines relatively to the silicon substrate, as a function of the horizontal distance from grating edge. This height sums up the residual thicknesses and the pattern imprinted depths at each measurement position.

These results also provide a very useful insight on the resist flow during NIL process. In this particular case, an analysis not detailed here shows that, at this scale, the horizontal resist flow is negligible in these experimental conditions. Finally, this example shows that scatterometry enables to go deeper into nano-rheology understanding as well as into the mechanical behavior of the mold during the imprint process. 


\section{Conclusion}

In response to the need of high-performance metrology for residual thickness measurement in nanoimprint lithography, the work presented here assess and show the interest of scatterometry, which is known to present numerous advantages. Scatterometry, as a non destructive way of measuring the residual thickness, appears to be a very promising tool for the optimization of the imprint process. We have shown that this technique is able to provide fast and accurate measurement for $\mathrm{NIL}$ as it has already been proved in $\mathrm{CD}$ metrology. Scatterometry is very well adapted for the measurement of residual layer thickness and provides very accurate results. Scatterometry is not a direct method; nevertheless the value of the residual error allows a first order assessment of the result quality. Moreover, scatterometry eases time consuming studies like the residual thickness measurement across a grating, and provides useful information on mold bending and resist behavior. We are presently using this tool to go deeper into these studies. We are also extending our scatterometric measurement capability to $3 \mathrm{D}$-features. 


\section{Figure caption}

Figure 1: Comparison between experimental (grey solid lines) and computed (black dotted lines with squares and triangles for 1-slice and trapezoidal 5-slice models respectively) scatterometric signatures, using 31-energy spectra (ranging from $1.5 \mathrm{eV}$ to $4.5 \mathrm{eV}$ with $0.1 \mathrm{eV}$ step), for (a) $350 \mathrm{~nm}$, (b) 500nm, (c) $1000 \mathrm{~nm}$, and (d) $1500 \mathrm{~nm}$ nominal line-widths (nested $\mathrm{L}: \mathrm{S}=1: 1$ lines) and using the 1-slice "square" and 5-slice "trapezoid" models for computation. The corresponding views show the superimposition of the SEM-cross sections with the scatterometric results using the "square" model.

Figure 2: Measurement precision (NMSE) as a function residual resist thickness for (a) $350 \mathrm{~nm}$, (b) $500 \mathrm{~nm}$, and (c) $1000 \mathrm{~nm}$, nested lines (L:S=1:1). The minimum of the curve corresponds to the best match between experimental and modeled features, using a "square" model.

Figure 3: Residual thickness measurements of the $\mathrm{L}=\mathrm{S}=350 \mathrm{~nm}$ grating zone located at wafer center, scanning from edge to the grating center by $20 \mu \mathrm{m}$ step. The residual thickness (bottom curve) and residual thicknesses + imprinted depths (top curve) are plotted as a function of the distance from the grating edge. 
(a) $\mathbf{L}=\mathbf{S}=\mathbf{0 . 3 5} \mu \mathrm{m}$
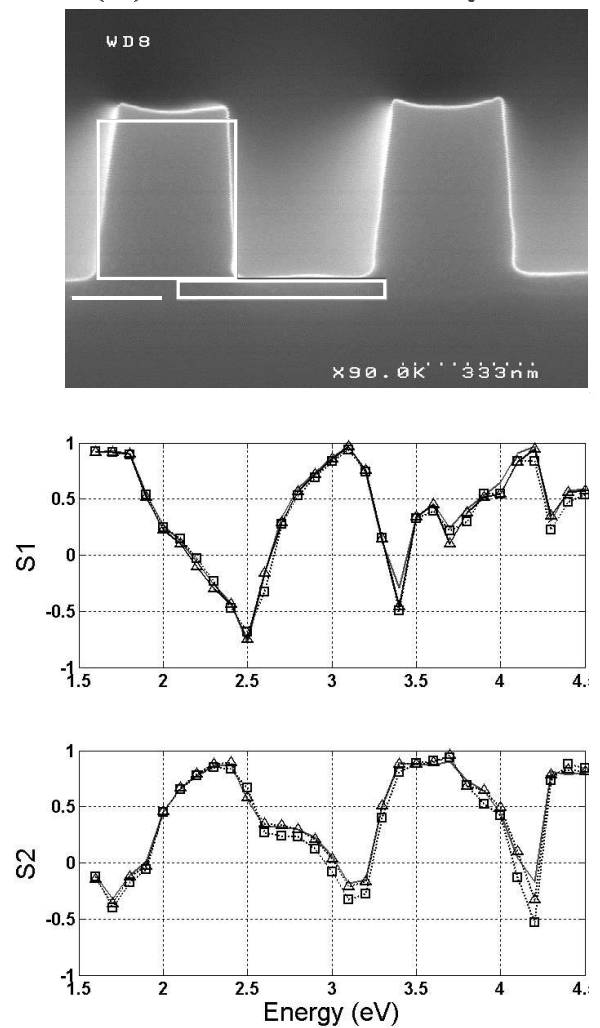

(c) $\mathrm{L}=\mathrm{S}=1 \mu \mathrm{m}$
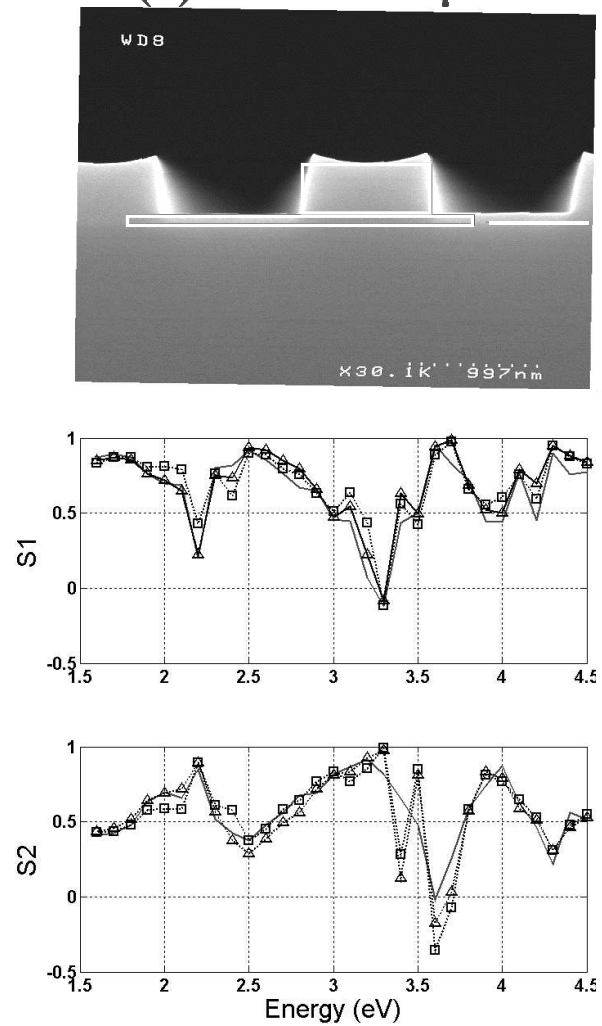

(b) $L=S=0.5 \mu m$
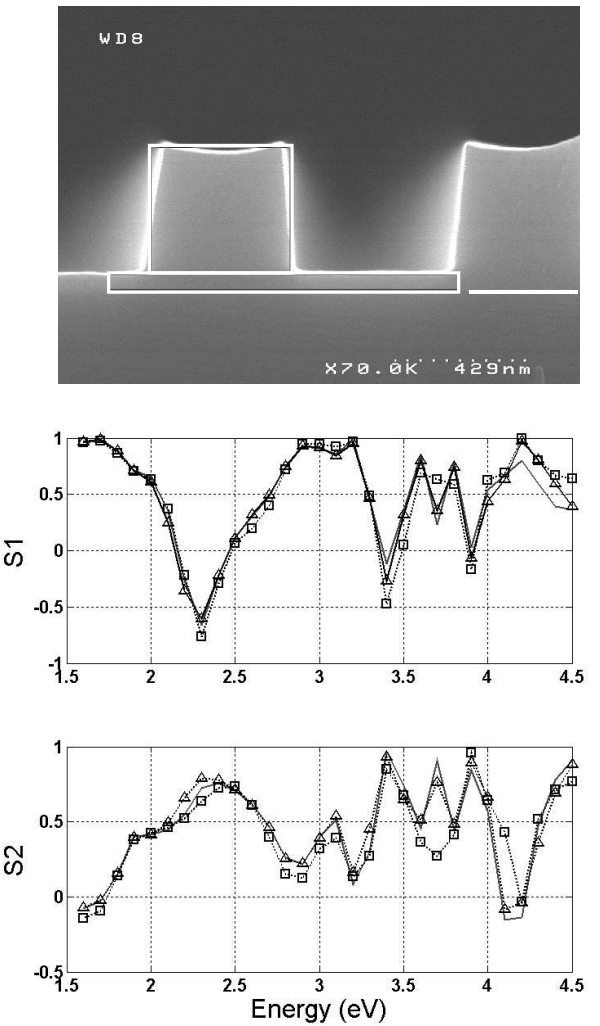

(d) $\mathrm{L}=\mathrm{S}=1.5 \mu \mathrm{m}$
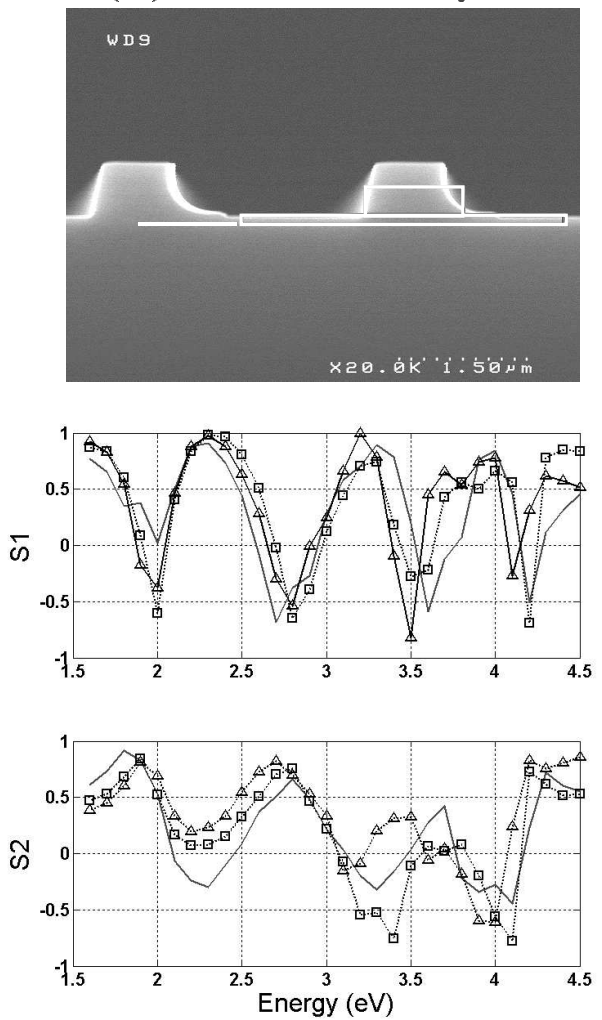

Figure 1 


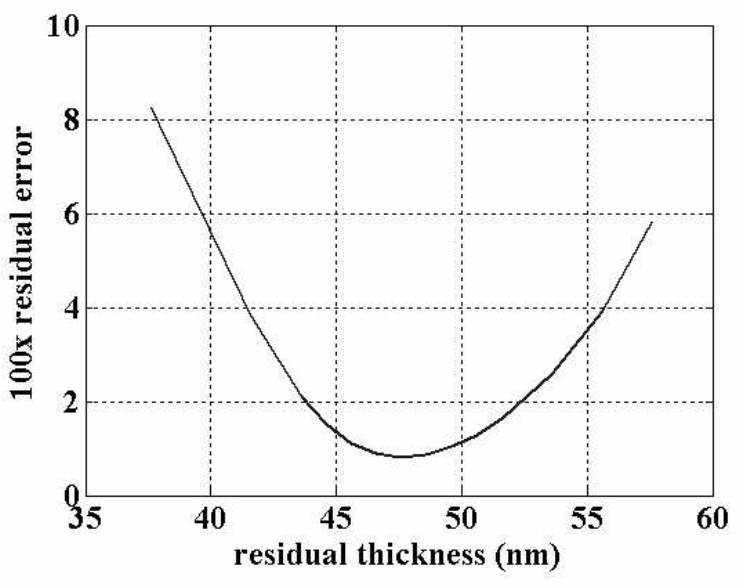

(a)

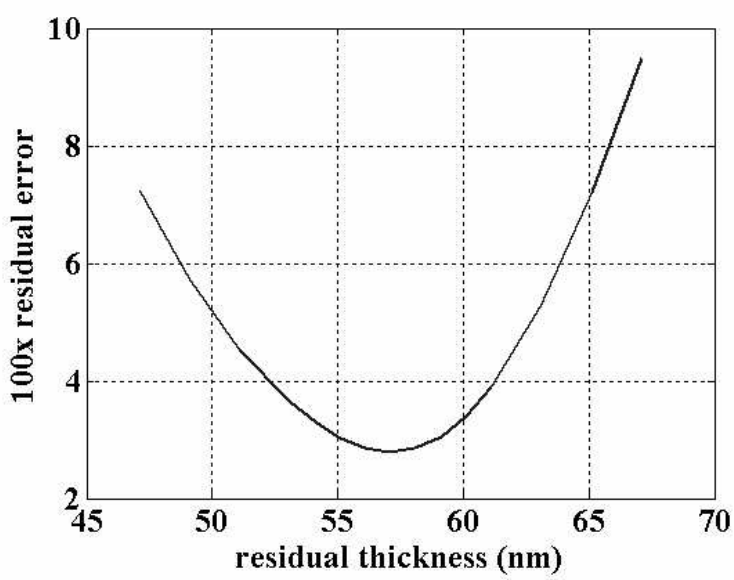

(b)

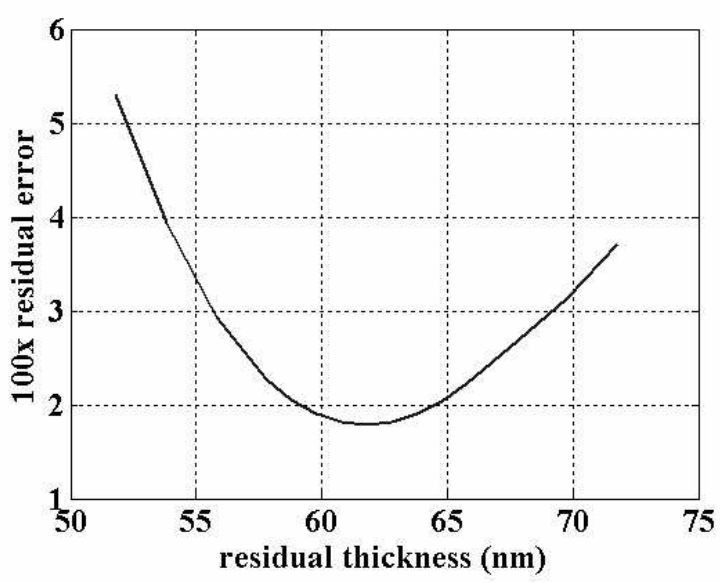

(c)

Figure 2 


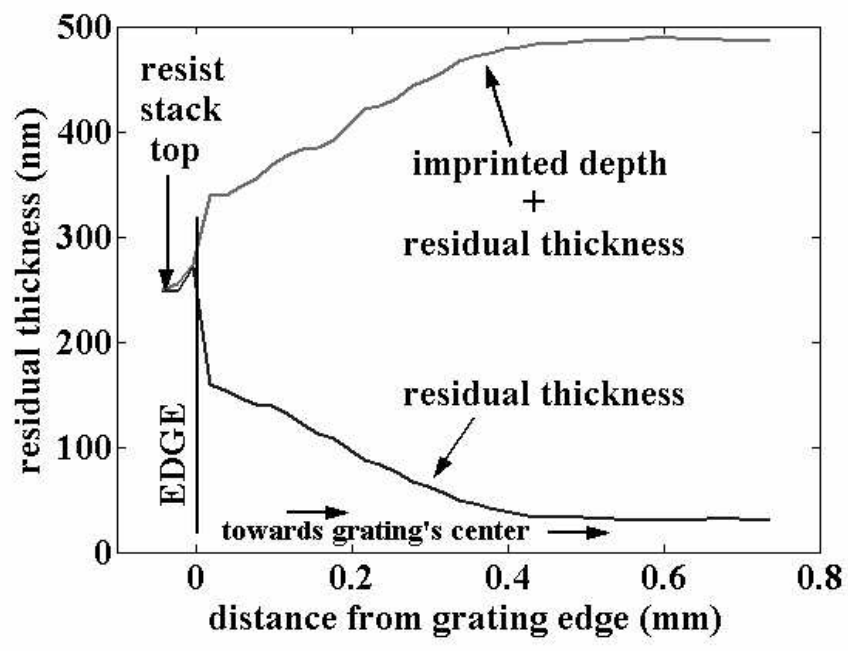

Figure 3 


\section{Table caption}

Table 1: Comparison between SEM-cross-section and scatterometric resist residual thickness (RRT) measurements. The residual error (error) and the computation time (CT) are given for each model used. The truncation orders (TO) used to compute scatterometric signatures are also reported for each feature.

Table 2: Additional information on feature characteristics, i.e. feature widths $\mathrm{FW}$, imprinted depths ID, and sidewall slopes SS, using both scatterometry and SEM-cs measurements. 


\begin{tabular}{lclllllll}
\hline \hline feature $1 / 2$ pitch & SEM-cs & \multicolumn{2}{c}{ "Square" model } & \multicolumn{5}{c}{ "Trapezoidal” model } \\
\hline (line = space) & RRT & RRT & Error & CT & RRT & error & CT & TO \\
\hline $350 \mathrm{~nm}$ & $\mathbf{4 9} \mathrm{nm}$ & $\mathbf{4 7 . 5} \mathrm{nm}$ & $0.81 .10^{-2}$ & $43 \mathrm{~s}$ & $\mathbf{4 7 . 8} \mathrm{nm}$ & $0.21 .10^{-2}$ & 150 & 7 \\
$500 \mathrm{~nm}$ & $\mathbf{6 1} \mathrm{nm}$ & $\mathbf{5 7 . 6} \mathrm{nm}$ & $2.8 .10^{-2}$ & $66 \mathrm{~s}$ & $\mathbf{5 9 . 3} \mathrm{nm}$ & $0.57 .10^{-2}$ & 341 & 9 \\
$1000 \mathrm{~nm}$ & $\mathbf{6 7} \mathrm{nm}$ & $\mathbf{6 0 . 3} \mathrm{nm}$ & $1.8 .10^{-2}$ & $156 \mathrm{~s}$ & $\mathbf{5 9 . 7} \mathrm{nm}$ & $1.4 .10^{-2}$ & 357 & 11 \\
$1500 \mathrm{~nm}$ & $\mathbf{6 7} \mathrm{nm}$ & $\mathbf{1 1 9} \mathrm{nm}$ & $9.9 .10^{-2}$ & $136 \mathrm{~s}$ & $\mathbf{1 3 5} \mathrm{nm}$ & $16.5 .10^{-2}$ & 244 & 11 \\
\hline \hline
\end{tabular}

Table 1 


\begin{tabular}{|c|c|c|c|c|c|c|c|c|}
\hline \multirow{2}{*}{$\begin{array}{l}\text { feature } 1 / 2 \text { pitch } \\
(\text { line }=\text { space })\end{array}$} & \multicolumn{3}{|l|}{ SEM-cs } & \multicolumn{2}{|c|}{ "Square" model } & \multicolumn{3}{|c|}{ "Trapezoidal" model } \\
\hline & FW & ID & SS & FW & $\mathrm{ID}$ & FW & ID & SS \\
\hline $350 \mathrm{~nm}$ & $310 \mathrm{~nm}$ & $410 \mathrm{~nm}$ & $6.2^{\circ}$ & $342 \mathrm{~nm}$ & $416 \mathrm{~nm}$ & $351 \mathrm{~nm}$ & $410 \mathrm{~nm}$ & $6.2^{\circ}$ \\
\hline $500 \mathrm{~nm}$ & $430 \mathrm{~nm}$ & $420 \mathrm{~nm}$ & $7.8^{\circ}$ & $447 \mathrm{~nm}$ & $430 \mathrm{~nm}$ & $461 \mathrm{~nm}$ & $420 \mathrm{~nm}$ & $7.4^{\circ}$ \\
\hline $1000 \mathrm{~nm}$ & $890 \mathrm{~nm}$ & $440 \mathrm{~nm}$ & $9.8^{\circ}$ & $944 \mathrm{~nm}$ & $412 \mathrm{~nm}$ & $951 \mathrm{~nm}$ & $410 \mathrm{~nm}$ & $6.3^{\circ}$ \\
\hline $1500 \mathrm{~nm}$ & unfilled & $610 \mathrm{~nm}$ & - & $1120 \mathrm{~nm}$ & $327 \mathrm{~nm}$ & $1126 \mathrm{~nm}$ & $326 \mathrm{~nm}$ & $10.3^{\circ}$ \\
\hline
\end{tabular}

Table 2 


\section{References}

${ }^{1}$ D. Fuard, O. Joubert, L. Vallier, and M. Bonvalot, J. Vac. Sci. Technol. B 19, 447 (2001).

${ }^{2}$ C. Gourgon, J. H. Tortai, F. Lazzarino, C. Perret, G. Micouin, S. Landis and O. Joubert, J. Vac. Sci. Technol. B 22, 602 (2004).

${ }^{3}$ The International Technology Roadmap for Semiconductors (Semiconductor Industry Association, metrology part, San Jose, 2004).

${ }^{4}$ C.J. Raymond, M.R. Murnane, S.L. Prins, S. Sohail, H. Naqvi, J.R. McNeil and J.W. Hosch, J. Vac. Sci. Technol. B 15, 361 (1997).

${ }^{5}$ P. Schiavone, C. Gourgon, V. Farys, N. Chaix, D. Fuard, "Measurement of residual thickness in nano-imprint lithography using scatterometry", NNT'04 Conference, Vienna (2004).

${ }^{6}$ M. G. Moharam, T. K. Gaylord, G. T. Sincerbox, H. Werlich, and B. Yung, Appl. Opt. 23, 3214 (1984).

${ }^{7}$ M. R. Murnane, C. J. Raymond, Z. R. Hatab, S. S. H. Naqvi, and J. R. McNeil, Integrated Circuit Metrology, Inspection and Process Control VIII, Proc. SPIE 2196, 47 (1994).

${ }^{8}$ S. S. H. Naqvi et al., J. Opt. Soc. Am. A 11, 2485 (1994).

${ }^{9}$ S. S. H. Naqvi, J. R. McNeil, R. H. Krukar, and K. P. Bishop, Microlithography World 2 (1993).

${ }^{10}$ G. Granet and B. Guizal, J. Opt. Soc. Am. A13, 779 (1996)

${ }^{11}$ S.A. Coulombe, B.K. Minhas, and C.J. Raymond, J. Vac. Sci. Technol. B 16, 80 (1998).

${ }^{12}$ K. Levenberg, Quarterly Applied Math. 2, 164 (1944).

${ }^{13}$ D. Marquardt, SIAM Journal Applied Math. 11, 431 (1963).

${ }^{14}$ H. Schift, L. J. Heyderman, M. Auf der Maur, and J.Gobrecht, Nanotechnology 12, 173 (2001).

${ }^{15}$ F. Lazzarino, C. Gourgon, P. Schiavone, and C. Perret, J. Vac. Sci. Technol. B 22, 3318 (2004).

${ }^{16}$ C.J. Raymond, M.R. Murnane, S. Sohail, H. Naqvi, and J.R. McNeil, J. Vac. Sci. Technol. B 13, 1484 (1995). 